\title{
Cultural evolution, energy use and human development
}

\author{
C. Viniegra \\ LawSchool, Universidad Panamericana, Mexico
}

\begin{abstract}
This analysis makes reference to life's biological laws and the biophysical boundaries to which all living beings are constrained in order to propose a theoretical model that links restriction and capacity to evolutionary strategies. The latter is related to cultural evolution, which identifies human culture as a phenomenon that stays within life's general evolution. After the model is presented, a statistical analysis is used for validation. Despite great diversity of cultural and historical backgrounds in present societies, data from economic, energy and technological features of more than 100 countries show striking regularities related to the way that physical and social resources are applied to the process of economic and human development, and show consistency with the proposed strategies of the model (Status Quo, Survival, Transformation and Growth). Among the most striking features discovered by this analysis, it is worth noticing that, apparently, there is a minimal threshold of energy expenditure and Research and Development (R\&D) investment required to achieve a high level of social development. Furthermore, in order to achieve a positive and strong cooperative effect between energy and R\&D, worldwide evolution of such indexes seem to indicate that economic and social evolution are constrained to similar scaling laws than those discovered for biological species.
\end{abstract}

Keywords: cultural evolution, economics, scaling laws, negentropy, energy use, life's laws.

\section{Introduction}

While the modern era has been a time of advances of every kind, human beings still face the persistence of ancient problems such as curable diseases, social 
marginalization and wars. At the same time, humankind faces new challenges because of the deterioration of the environment, changes in climate conditions of the planet, exhaustion of key resources such as farming fields and clean water, along with the growth of social pathologies such as addictions and new violence phenomena that range from the family to the work environment. When thinking about old and new issues, decades of strategies aimed to solve them have accumulated and results are the opposite of what was expected and the provision of ideas seems insufficient to explain or solve even one important issue at its root.

Due to the recurrence of crisis of all types and to the probable perspective of disasters, such as a global climate change that can create, among other things, hunger and massive migration of people, it has become necessary to review our understanding of how human culture develops and relates to general evolution and use of the environment.

\section{Life's laws and the human being}

Life is the process in which a finite amount of matter self-organizes, metabolizes (uses energy from the environment), grows, reproduces and evolves [1].

\subsection{Self-organization, metabolism and growth}

According to the second law of thermodynamics, life generates higher levels of order [2] by importing negative entropy for itself, also known as negentropy or syntropy, while exporting a larger amount of entropy to the environment. In the aforementioned thermodynamic exchange, life increases order in a process called data exchange [3]. In this way, energy obtained from the environment is used through metabolism to organize molecules in coherent sequences of information, known as genetic information. The byproduct of this process is heat release or the exchange of entropy to the environment.

Generation of higher levels of order or information exchange require energy use and dissipation of heat. In this process, the cells of all living organisms are geared to increase the successful occupation of an ecological niche with a minimum of energy expenditure and seem to be bounded by the laws of mass and energy transfer at a microscopic scale since all of them have very large surface-to-volume ratios in order to minimize local concentration and temperature gradients. Also, large multicellular organisms need vascular systems in order to distribute nutrients and oxygen in an efficient way. This has been reflected in a universal mathematical rule called Kleiber's Allometric Law of Scaling [4].

\subsection{Reproduction}

Living beings deteriorate over time and, with deterioration, life eventually disappears. Nonetheless, the phenomenon of life, through the genetic mechanisms of reproduction, inherits an updated copy of its genetic information to a new being whose entropy is low enabling the continuity of the process of 
life. When defining the guidelines by which life operates, the first one is that in spite of being circumscribed to the universal laws of physics, life uses everything within its reach to be continuous through time.

In the reproduction process of living beings, two different strategies can be observed, explained under the " $\mathrm{r} / \mathrm{K}$ Selection Theory" [5].

Living beings oriented towards an " $r$ " reproduction strategy exist in unstable environments and non-saturated ecological niches where high speed of reproduction is crucial for survival. The characteristic features on this strategy are low energy investment to the descendants and high fecundity, low body volume, early maturity, short life expectancy and wide dispersal of the population. Rodents are a mammal example. Scientific literature points " $r$ " selected species as opportunists.

Living beings oriented towards a " $\mathrm{K}$ " reproduction strategy exist in stable environments and niches close to their maximum load capacity; their fecundity rate is low, energy investment from parents to descendants is high, maturity is slow and life expectancy is long. Big mammals are the main examples of species under "K" selection. Scientific literature points "K" selection species as balance species.

\subsection{Evolution}

The physical margins where life is possible seem small, but there is enough room within them to generate adaptation needs [6]. These are covered in the genetic traits that persist or get discarded through the reproduction process. Regardless of the fact that the most popular interpretation on Darwin's proposition, "survival of the fittest", is survival of the biggest, strongest, and fiercest, a better interpretation on Darwin's work is that fitness should be understood as the selection of genetic features that guarantee, in the long run, the survival of the best adapted [7].

As an example, it can be said that in similar environments, discounting energy availability, living beings with high metabolic rates will be better adapted when energy is abundant and, given that abundance, it may be necessary to compete in a numerous population. On the contrary, in an environment with low energy availability, living beings with low metabolic rates and greater willingness to collaborate will be more successful [8].

\subsection{Restriction and capacity}

Life is limited by nature's laws, but given the opportunity, it widens its capacities to accumulate negentropy and responds to environmental changes using the evolution mechanism. In this light, one may conclude that there are two working axes that determine the success or failure of the changes and their persistence through time.

\subsubsection{Restriction - the environment, the external}

The list of environmental variables that affect living beings is long, but the most bounding resource is energy. For example for autotrophic organisms (mainly 
photosynthetic) the main constraint is solar energy transformed in biomass. For heterotrophic organisms, living out of autotrophic organisms, added to the previous limitation, the conversion efficiency is roughly $10 \%$ for each trophic level under consideration (herbivores, carnivores and detrivores). The fact, that life occurs in a limited space, with limited energy of solar input, makes obvious that life is bounded by spatial and energy constraints.

\subsubsection{Capacity - what is of each, the intrinsic}

With life's existence, in spite of the higher transfer rate of entropy towards the universe, a greater order is produced in its operating range. In the long term and as a second directive of life, life works to widen its possibilities of information exchange or negentropy accumulation. At the limit, life will achieve as much order as the available energy and entropy level of the environment will allow.

The dependence on the environment and its changes produce exchanges among the time permanence directive versus the capacity to generate negentropy (negentropic capacity) directive. The latter prevents that the speed in which life spreads through the environment to be constant since changes in the environment and restriction levels define the necessity to modulate the evolution process in order to guarantee permanence in time.

The acceleration in capacity growth requires larger amounts of energy, either due to a population increase or to an increase in the complexity of the living beings that are part of the process. However, in spite of the irrevocable calling of life to generate as much order as possible, the speed to do it will vary in time as a function of the acceleration and deceleration of the capacity to generate negentropy.

\subsection{Metabolic model}

This paper has described the features of living beings, biological and physical laws that limit them and two axes of action, restriction and capacity. In that light, and using the environment as the external and capacity as the internal or what is intrinsic to life, it is possible to propose an analysis of the gathered elements in a model generated by the metabolic strategies produced by intersecting restriction and capacity (see Figure 1).

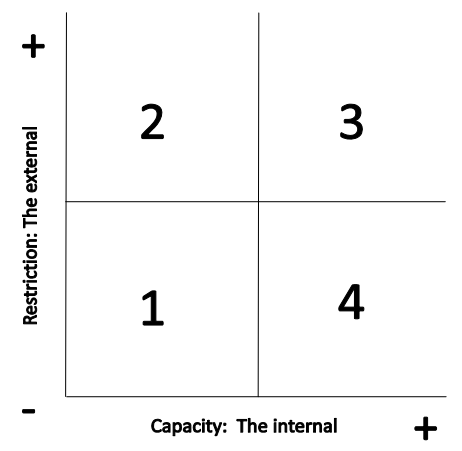

Figure 1: $\quad$ Schematic representation of the model. 
Quadrant 1. Low restriction/Low capacity: Status Quo. On these coordinates, available resources supplied by the environment surpass the metabolic needs of the population. The environment is unstable either by reproductive competition and/or environmental variations. Additionally, there is no change in the capacity to generate negentropy. The reproductive rate is maximized but given the low negentropic capacity of the population, the resources' consumption rate is lower than the environment's replacement rate.

Quadrant 2. High restriction/Low capacity: Survival. On this quadrant, the energy availability is compromised and there are no chances for expansion due to the impossibility to increase negentropic capacity. In metabolic terms, all energetic non-essential pathways disappear and energy use rate is minimized. If the environment's replacement rate is overrun, there is a reduction of population or death.

Quadrant 3. High restriction/High capacity: Transformation. The shortage of resources is compensated by an accelerated growth in negentropic capacity to take on new opportunities. There are potential but not present threats; every new function is enabled to seize a new opportunity within the environment.

Quadrant 4. Low restriction/High capacity: Growth. High availability of resources allows the colonization of the environment. It is possible to increase the negentropic capacity and the metabolic rate at the same time. Competition is produced by the population's increase and metabolisms that consume more energy are favoured.

\subsection{Hemispherical analysis of the model}

By dividing the plane into four sections with two lines one vertical and another horizontal, the resulting hemispheres will be: vertical cut, left and right hemispheres; horizontal cut, top and bottom hemispheres.

\section{Vertical}

- Left. Low evolution and capacity. Environmental conditions overcome life's long term evolutionary dynamics, options are limited. Negentropy generation is limited.

- Right. High evolution and capacity. The long term dynamic for capacity growth, works unbounded in any environmental conditions. Negentropy's generation grows, as well as energy use and the exchanged entropy with the environment.

\section{Horizontal}

- Top. There is little waste of energy, cooperation prevails over competition. The challenge imposed by the environment is more important than rivalry, thereby metabolism is optimized. In addition, efficiency levels used to incorporate energy to life process are increased. On the reproductive side, selection is oriented towards " $\mathrm{K}$ "; there is low variation in environmental factors and low rivalry level. 
- Bottom. Energy use is maximized; competition prevails over cooperation; reproductive competition is more important than the environment's challenge. Efficiency is not important, but final metabolic capacity is. Reproductive selection is oriented towards " $r$ "; there is instability due to environmental factors and/or because a high degree of rivalry.

\subsection{Ecological niches}

To enhance the analysis it is also important to make reference to different types of living beings and the ecological niches they hold within the general model while explaining Figure 2.

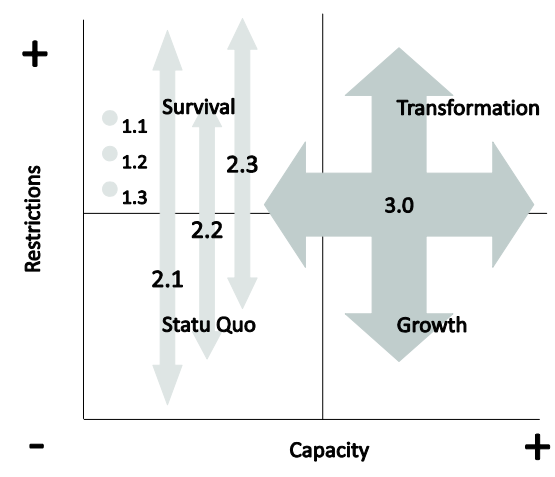

Figure 2: $\quad$ Ecological niches.

Autotroph. They populate the different restriction levels by differentiation of species, there is no locomotion or behaviour; in Figure 2 a species is a point, the whole forms a negentropic capacity line (photosynthesis). They evolve through physical features or "hardware". (Points: 1.1, 1.2, 1.3 in Figure 2.)

Non human Heterotrophs. They acquire the capacity so that each species populates a vertical line and, through locomotion, they generate a new adaptation strategy: migration, which means capacity to run away from danger, attack, pursue pleasure (instinct); behaviour emerges and is always closely related to "hardware" configuration. Species manage to settle on new capacity spaces by differentiating their energy strategy: herbivorous, carnivorous, and omnivorous. They also evolve through "hardware". Locomotion accelerates evolutionary competition. (Lines 2.1, 2.2, 2.3 in Figure 2.)

Human Heterotrophs. They abandon evolution by "hardware" (physical features) and represent a new evolutionary strategy for life; evolution through "software". They are capable of choosing any quadrant, be it by migration, aversion to pain, attraction to pleasure and by cultural development. They have the ability to choose their behaviour, which adds extra capacity mechanisms and information to the ones granted by genetic inheritance. They manage to change the pattern of resource usage in a quick way through a new mechanism, cultural evolution [9] that is inherited to their descendants through knowledge 
transmission (cultural reproduction) which means the accumulation of negentropy in culture. (Arrows 3.0 in Figure 2.)

\subsection{Cultural evolution}

In biological terms, the human being is close to the extreme of " $\mathrm{K}$ " selection within the reproduction strategy. This means that it improves his descendants' survival possibilities by investing in them a great amount of energy. " $\mathrm{K}$ " selection also means there is a long maturity period for individuals and a longer life span.

Unlike some species under " $K$ " selection, such as elephants and whales, the human being does not have great body volume or exceptional physical capacities, but it consumes huge amounts of energy. As stated before, the main difference in man's survival strategy lies within the reduction of physical and genetic competition with other species in order to evolve quickly through a new strategy already defined as cultural evolution.

Exactly like genetic evolution, cultural evolution generates negentropy through the use of energy exchanged for higher levels of information expressed in knowledge, art and technology. Furthermore, just as it happens on genetic evolution, new levels of information (knowledge) generate new possibilities to access energy sources and for new ways to make use of the environment (technology). The latter is done in a way similar to what happened when energy strategies like the herbivorous, the carnivorous, and the omnivorous appeared. In philosophy, a rise in the understanding of the universe is defined as an increase in the consciousness level or approximation to the truth. In biology, the difference between conscious (man) and unconscious beings (the others) is also pointed out.

Even though the theme of consciousness is one of the most debated points in religious theology and secular philosophy, both approaches point at the need to answer the main ontological and cosmological questions: What exists? What is life for? Is the universe infinite? Does time have a beginning and an end? What is the essence of matter? It may be presumed that whoever manages to obtain an answer to any of these questions would be on a higher level of consciousness that those who do not.

Moreover, making definitions based on features that separate unconscious animals from conscious man has become complicated since ethology reports more complex behaviours in animals than the ones assumed before (i.e. the chimpanzee's use of tools, cooperation actions, learning and problem solving within various species), while neurosciences acquire better understanding of the human brain and report higher intervention of the subconscious in people's actions and motives than ever imagined [10].

Therefore, it is possible to say that the gradient going from the inert to the subconscious and to the conscious, is related to the non living, life and life's level of organization according to the capacity to generate negentropy. This positively correlates energy use and the level of consciousness, where the effective use of energy (energy efficiency), is more important than total use of it. 


\subsection{Energy and human development}

Figure 3 shows the relationship for 126 countries, between the Human Development Index (HDI) and per capita energy consumption $(\mathrm{ToE}=$ Tons of oil Equivalent) and the best adjusted curve seems to be a logarithmic model. It is worth noticing that all countries with HDI value higher than 0.9 use more than $2 \mathrm{ToE}$ per capita. Also, all countries with per capita consumption lower than 0.7 ToE have an average HDI of 0.6. The corresponding empirical equation can be written as follows:

$$
\mathrm{HDI}=\mathrm{k} \ln \left(\mathrm{ToE} / \mathrm{ToE}_{0}\right)+\mathrm{B}
$$

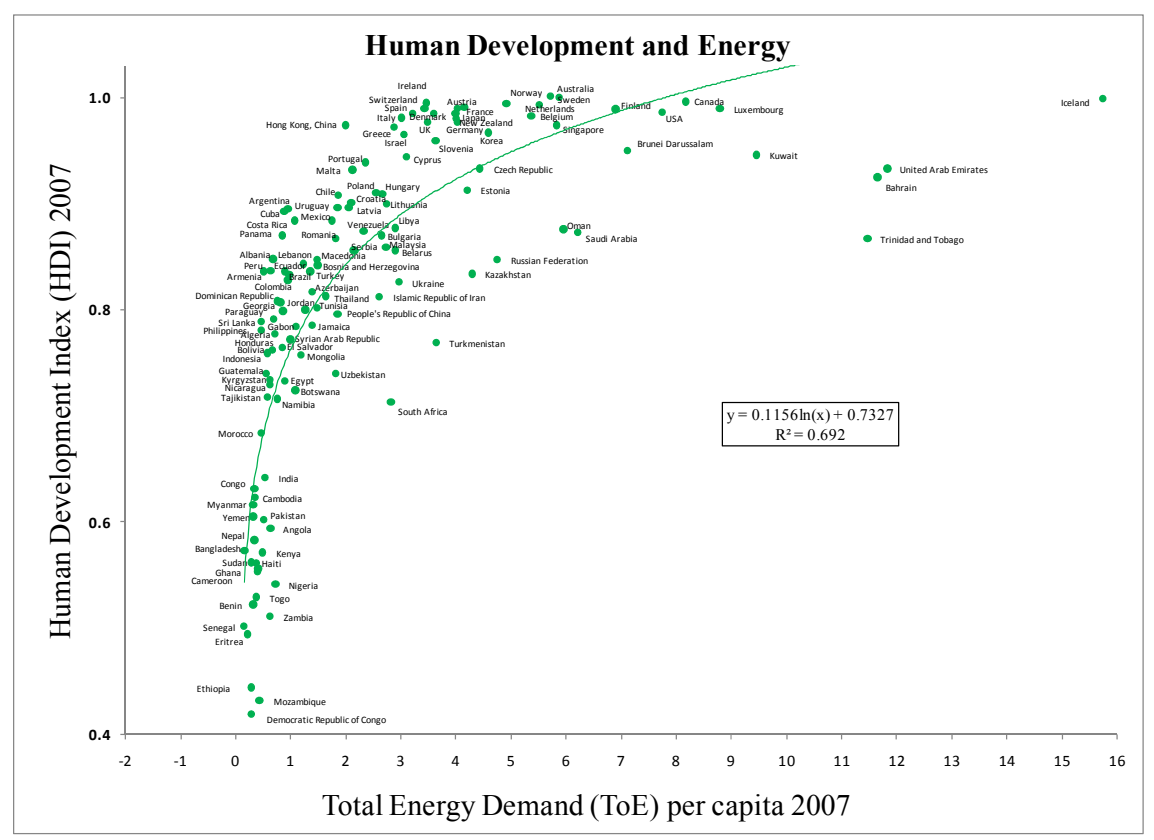

Figure 3: Human development and energy demand. Source: HDI, United Nations Development Programme (www.undp.org), total energy demand, International Energy Agency (www.iea.org). ToE is a standardized unit equal to the amount of energy obtained through the combustion of a ton of crude oil. It is equal to 41.868 Giga Joules and to 11.63 Mega Watts/hour.

Expression (1) has a threshold value when $\mathrm{ToE}=\mathrm{ToE}_{0}=1$, and, $\mathrm{HDI}=\mathrm{B}=$ 0.737 , beyond which, the values of ToE increase very fast in order to approach the highest level of human welfare with per capita values of ToE higher than 3 . The value of the empirical constant, $\mathrm{k}=0.1156$, seems to be a global efficiency factor, since the correlation shown in Figure 3 was taken from a sample of 126 countries (more than $90 \%$ of human population) and would be related to an intrinsic property of present human societies. 
This is a very important consideration in terms of planning the future because it implies that the improvement of the quality of life for nearly 5 billion people, living with HDI lower than 0.7 , will require a very large increase of global energy expenditure. Otherwise, the social and political tensions across the world will increase breaking down the present level of international agreements. For example, terrorism and widespread violence will be difficult to eradicate in a world where most of the people experience a very low HDI environment.

\subsection{Energy and technology development}

We can state that total energy use of a society $\mathrm{E}_{\mathrm{T}}$ has three terms

$$
\mathrm{E}_{\mathrm{T}}=\mathrm{E}_{\mathrm{M}}+\mathrm{E}_{\mathrm{G}}+\mathrm{E}_{\mathrm{RD}}
$$

where $E_{M}$ is the energy required for maintenance, $E_{G}$ is the energy required for growth and $E_{R D}$ is the energy to be invested in $R \& D$ (capacity growth). Here it is not intended to estimate each fraction of the total energy budget. Instead it is assumed that there should be a power law between $\mathrm{E}_{\mathrm{RD}}$ and $\mathrm{E}_{\mathrm{T}}$ and we also assume that energy expenditure is a reflection of the technological capacity of each society, measured as a normalized innovation index, $\mathrm{I}_{\mathrm{N}}$, defined as the number of patents registered in the US Patent Office each year and assigned to nationals of each given country and normalized per million people. This index has two interesting properties; it involves the largest patent registrar in the world where international companies compete for the largest share in the US market, and also, it only takes into account the patents where the assignee (the owner of the patent rights) is from a given country. This way, the innovation index is strongly related to innovation supported by companies or individuals that wish to risk money in patent registration expenditures and have residence in a given country. The power law is of the following form

$$
\mathrm{E}_{\mathrm{T}}=\mathrm{E}_{\mathrm{T} 0}\left(\mathrm{I}_{\mathrm{N}} / \mathrm{I}_{\mathrm{N} 0}\right)^{\mathrm{n}}
$$

Figure 4 shows the log-log correlation implied in the power law between ToE and $I_{N}$; it should be noted that from the previous list of 126 countries, only 74 have patents assigned to individuals or companies resident in those countries. It is worth noticing that the variation coefficient is only $\mathrm{R}^{2}=0.51$, suggesting that the total energy expenditures related to technological development require a more careful partitioning. But it shows a general trend that may help to establish worldwide country typology in the way that energy expenditures are related to innovation. This preliminary model of energy expenditure follows the empirical equation

$$
\mathrm{ToE}=2.1394 \mathrm{I}_{\mathrm{N}}^{0.2108}
$$

In Figure 4 it can also be seen that the variable defined to evaluate capacity (patents/million inhabitants) increases through various magnitude orders that range from less than 0.1 patents a year per million inhabitants, up to more than 200. This way, developing countries are clearly separated from developed ones. It is worth noting that there is not a single case of a developing country with low energy consumption and high patents' registration rate. 


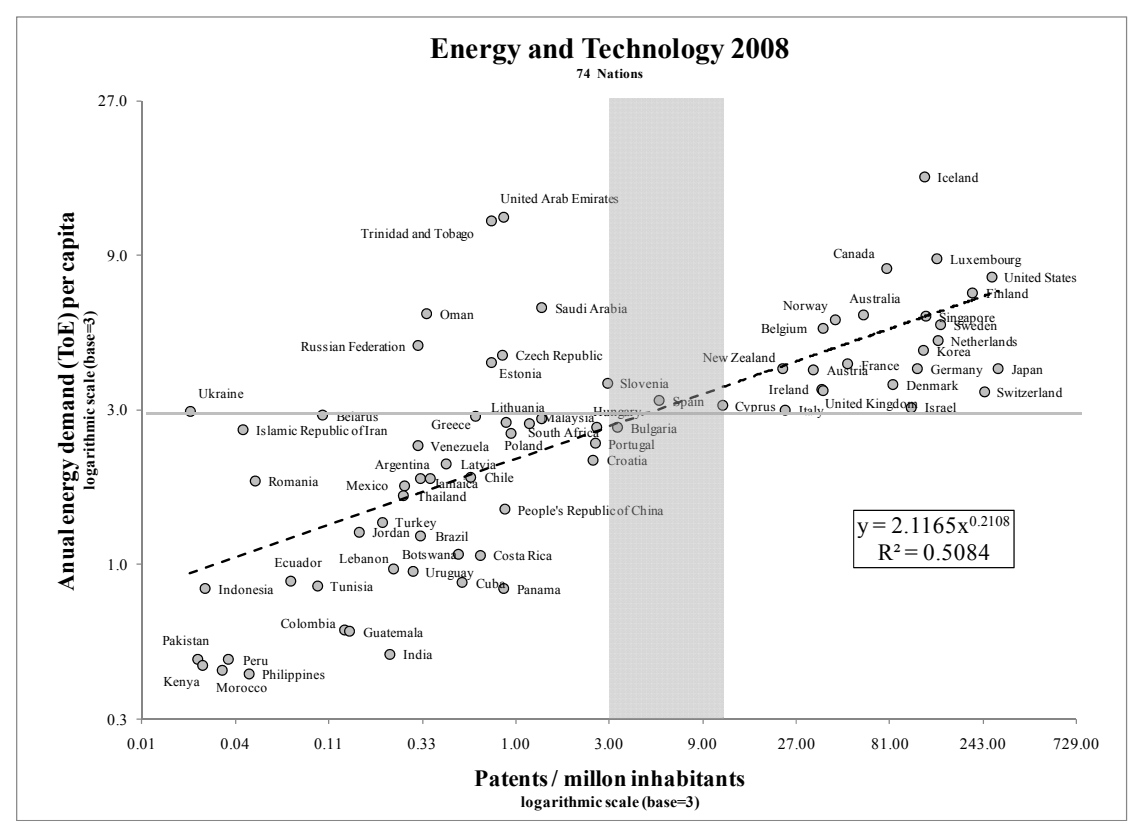

Figure 4: Energy and technology. Source: energy International Energy Agency (www.iea.org) and patents from the U.S. Patent Office (www.uspto.gov).

Again this seems to indicate the existence of threshold around $I_{N}=3$ patents per million people, as a requisite to reach an energy expenditure, per capita, of 3 ToE. In Figure 4 there is a shadowed region that covers from 3 to 10 patents a year per million inhabitants and seems to be the transition zone between development scales and contains countries under change process, from underdevelopment to development.

The slope of the empirical power equation in Figure 4 may be used as an indication of the comparative efficiency reciprocal interaction between energy expenditures and technological development. There are some interesting cases to discuss. For example, the United Arab Emirates, Trinidad and Tobago, several oil exporter countries and some ex Soviet countries, have a value of $\mathrm{I}_{\mathrm{N}}$ lower than 0.33 patents per million although they have energy expenditures higher than 3 ToE per capita. However, they cannot be considered members of the highly developed nations club since their HDI is lower than 0.9.

The most suggestive aspects in Figure 4 are the empty spaces at the right bottom and left top quadrants. Empty spaces indicate possible hindrances or forbidden zones, top and bottom, of a scaling rule between energy use and development. In fact when all points $(2,115)$ from a data base with a data set from 1978 to 2007 were plotted (Figure 5) and analyzed with a potential model, the result was a regression equation equal to $\mathrm{y}=1.63 \mathrm{x}^{0.26}$, with a variation coefficient $\mathrm{R}^{2}=0.59$, where " $\mathrm{y}$ " is equal to primary energy supply in ToE units and " $\mathrm{x}$ " is equal to patents registered in year per million inhabitants. 


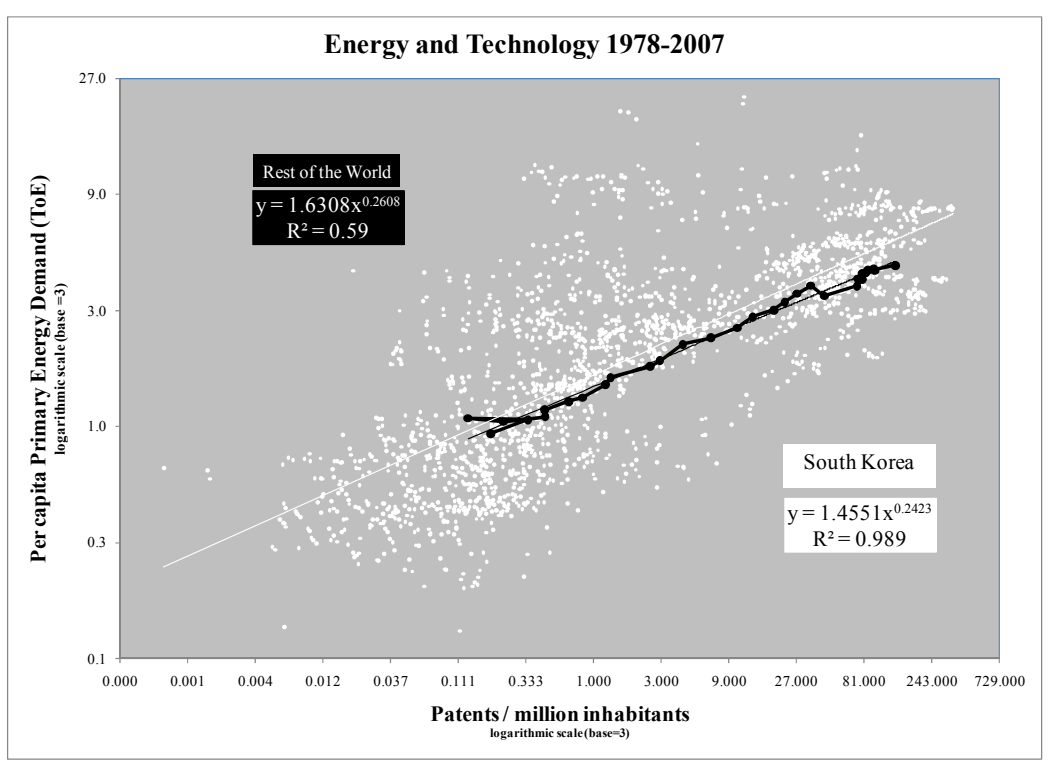

Figure 5: Evolution of energy and technology from 1978 to 2007. South Korean performance is indicated in black with a correlation function $\mathrm{y}=1.4551 \mathrm{x}^{0.2423}\left(\mathrm{R}^{2}=0.989\right)$. Source: energy International Energy Agency (www.iea.org) and patents from the U.S Patent Office (www.uspto.gov).

After making a diachronic (related to time) analysis with an online tool named "Motion Chart" from "Google Docs", it was oberved that that the nation with best performance, and the only one that advances several orders of magnitude in both axes is South Korea. A nation that frequently is referred in specialized publications as an example of success given the fast achievement in economic and social development. For this reason, a specific analysis was made only with South Korea's data, also presented in Figure 5, here the set of couples of values $\left(\mathrm{ToE}, \mathrm{I}_{\mathrm{N}}\right)$ were plotted vs. time and the regression analysis showed the following empirical equation

$$
\mathrm{ToE}=(1.45) \mathrm{I}_{\mathrm{N}}^{0.24},\left(\mathrm{R}^{2}=0.98\right),
$$

This is a striking result suggesting that this equation is indeed a scale-up equation between energy and technology and supports the notion that the power law of Figures 4 and 5, is the "developtmental pathway" for social and economic improvement. Here, the scaling coefficient is approximately " $1 / 4$ ".

Figure 6 shows the diacronic analysis of, ToE vs. $\mathrm{I}_{\mathrm{N}}$, for Costa Rica, Mexico, South Korea and Saudi Arabia. The only growing up curve belongs to South Korea. The other three cases oscilated as if they were caught in an under development trap. Curiously enough, the main numerical difference of those plots is not the slope (the power $1 / 4$ ) but the scale-up reference value $\mathrm{ToE}_{0}$. Apparently, this is related to a given choice of resource allocation. A high level 
of $\left(\mathrm{ToE}_{0}>1.45\right)$ would imply to much attention paid to energy expenditure with low level of technology development, as seems to be the case of Saudi Arabia. A low level of $\mathrm{ToE}_{0}$ would imply that there is a weak coupling between technological development and energy expenditures. And there is a third case, Mexico; here the trajectory is apparently superimposed to the ascending trajectory of South Korea but in fact, it has oscillated in approximately the same place of the phase plot during the last 30 years. This also may be related to the lack of long term commitment in order to establish a working link between technological development and energy expenditures. It is worth noticing that Mexican R\&D expenditures have been maintained at a level lower than $0.4 \%$ of GDP during the last three decades and the gap between scientific publication and technological innovation is well known.

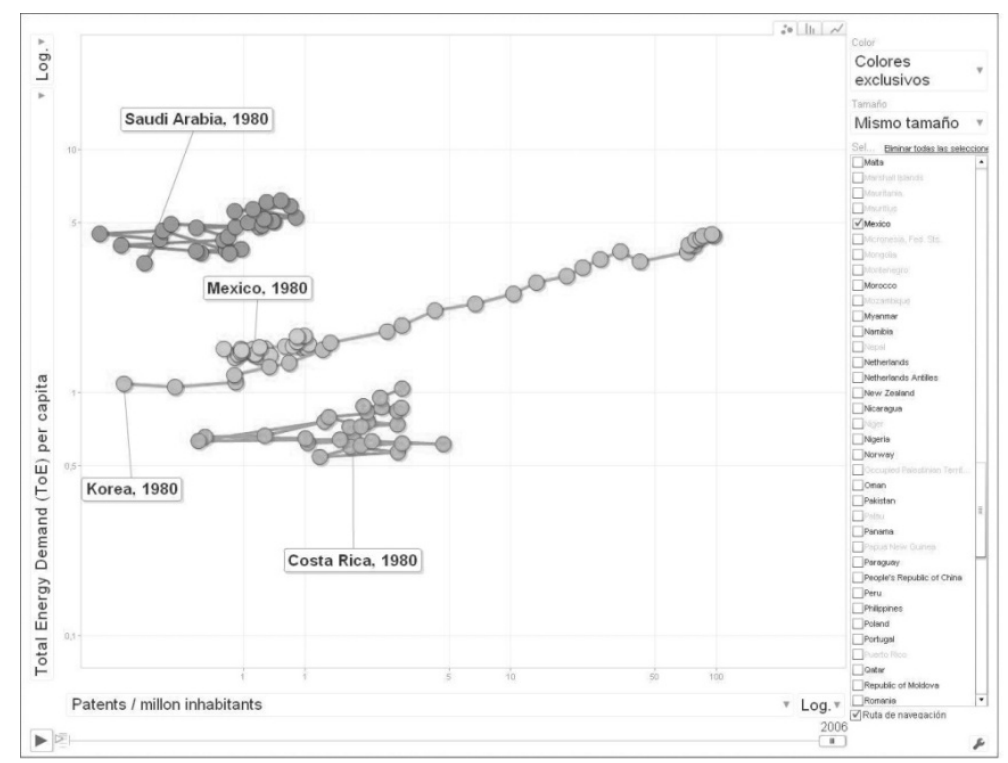

Figure 6: Diachronic analysis (selected countries). Diachronic analysis was made with "motion chart" from Google ${ }^{\circledR}$ docs (docs.google.com).

It is also worth noticing that the concepts "economy of scale", and "marginal analysis" used in economics, come from the same principle as the biologic scaling rules: large systems are more efficient, but the costs they generate, including externalities - at some stage - grow faster than the benefits they generate, to the point where obtained additional benefit is lower than the cost increase. An example of probable boundaries and efficiency differentials is observed in the position held by Switzerland and the United States (Table 1). It is necessary to highlight that in a closed system like the earth, the non inclusion of externalities to the overall development cost accumulates systemic risks in the form of a reduction of the biospheres capacity to regenerate resources, which 
means the consecution of the life expansion directive without balance with the permanence in time directive.

Table 1: $\quad$ Energy efficiency of Switzerland and the USA.

\begin{tabular}{|l|c|c|}
\hline \multicolumn{1}{|c|}{ Concept } & Switzerland & United States of America \\
\hline GDP per capita (USD) & $\mathbf{6 4 , 0 1 1}$ & $\mathbf{4 6 , 7 1 6}$ \\
\hline Energy demand (ToE) per capita & $\mathbf{3 . 4 2}$ & $\mathbf{7 . 7 4}$ \\
\hline Patents a year / million inhabitants & $\mathbf{2 4 6 . 0 7}$ & $\mathbf{2 6 7 . 6 1}$ \\
\hline Human development index & $\mathbf{. 9 6 0}$ & $\mathbf{. 9 5 6}$ \\
\hline
\end{tabular}

\section{Conclusions}

All living beings are found in any of four possible metabolic strategies due to the exchanges generated within the relationship between restriction and capacity.

When seen through the lens of energy use and exchange of information, human culture abides to the fundamental laws of life and can be considered to remain within life's general evolution. Given that human societies follow similar development pathways as the ones proposed on the model and when measured by their use of resources and by their technological output.

$>$ The self determination capacity humans have to design their own existence relates to the capacity to move to any of the four metabolic strategies. Thus, the cultural diversity of human kind can be understood, as the cultural evolution of human groups which have selected different survival strategies.

\section{References}

[1] Nielsen, P. E., A New Molecule of Life. Scientific American, December 2008.

[2] Rubí, M. J., Does Nature Break the Second Law of Thermodynamics? Scientific American, October 2008.

[3] Brillouin, L., Negentropy. Journal of Applied Physics, pp. 1152-1163, 1953.

[4] Kleiber, M., Body Size and Metabolic Rate. Physiological Reviews, 27, pp. 511-541, 1947.

[5] Pianka, E.R., On r and K selection. American Naturalist, 104, pp. 592-597, 1970.

[6] Darwin, C., The Origin of Species, John Murray: London, 1859.

[7] Shermer, M., Darwin Misunderstood. Scientific American, p. 34, February 2009.

[8] Kropotkin, P., Mutual Aid: A Factor of Evolution, Forgotten Books: London, (Conclusion), 1902.

[9] Teilhard de Chardin, P. The Phenomenon of Man, Evergreen Review, Kindle p. (Kindle location 429), 1955 (Kindle 2009)

[10] Zaltman, G., Marketing Metaphoria. Harvard Business Press: Boston, pp. 47-61, 2008. 